\title{
Discriminant Mutual Subspace Learning for Indoor and Outdoor Face Recognition
}

\author{
Zhifeng $\mathrm{Li}^{1,2}$, Dahua Lin ${ }^{2}$, Helen Meng ${ }^{1}$, and Xiaoou Tang ${ }^{2,3}$ \\ ${ }^{1}$ Dept. of SEEM, The Chinese University of Hong Kong \\ \{zfli, hmmeng\}@se.cuhk.edu.hk \\ ${ }^{2}$ Dept. of IE, The Chinese University of Hong Kong \\ \{zli0, dhlin4, xtang\}@ie.cuhk.edu.hk \\ ${ }^{3}$ Microsoft Research Asia, Beijing, China \\ xitang@microsoft.com
}

\begin{abstract}
Outdoor face recognition is among the most challenging problems for face recognition. In this paper, we develop a discriminant mutual subspace learning algorithm for indoor and outdoor face recognition. Unlike traditional algorithms using one subspace to model both indoor and outdoor face images, our algorithm simultaneously learn two related subspaces for indoor and outdoor images respectively thus can better model both. To further improve the recognition performance we develop a DMSL-based multiclassifier fusion framework on Gabor images using a new fusion method called adaptive informative fusion scheme. Experimental results clearly show that this framework can greatly enhance the recognition performance.
\end{abstract}

\section{Introduction}

Most current face recognition algorithms work well on dataset obtained under controlled lighting condition. However, the performance drops significantly for data collected under uncontrolled outdoor lighting condition. This is clearly shown in the face recognition vendor test 2002 (FRVT 2002) [1], where the best face recognition systems perform poorly on the outdoor probe face images.

A number of approaches have been proposed tackling the difficulty caused by varying illumination conditions. Most use the Lambertian reflectance model to describe the facial variations under different lighting conditions. Under the Lamberitian assumption, the set of images of one face lies in a 3D linear space, which can be reconstructed using three training samples taken under different lighting conditions [8]. Basri et. al. [9] proved that this image set can be approximated as a 9D linear space considering the attached shadows but ignoring the cast shadows. Georghiades et. al. [10] proposed an illumination cone with infinite di- mension accounting for attached shadows and cast shadows. However, under complicated outdoor conditions these approaches are not suitable. There are multiple light sources, and the reflectance from each lighting source will be significantly affected by the cast shadows caused by other objects. These produce complicated variations on the outdoor facial images and thus make them significantly different from the indoor facial images. Such great discrepancies between the indoor and outdoor facial images make it infeasible to use a simple model for their comparison.

Recently, Wang and Tang [2] showed that both the Gabor features [6] and unified subspace analysis [7][15] can reduce the lighting variation in face recognition and integrate them to solve the indoor and outdoor face recognition problem [2]. However, this method still uses a single subspace to describe both indoor and outdoor face images. In this paper, we develop a novel framework called discriminant mutual subspace learning (DMSL), in which two related subspaces are simultaneously learned to model the indoor and outdoor samples. An efficient scheme is derived for optimization through mutual interactions between the two subspaces. In addition, to further improve the recognition performance, we develop a DMSL-based multi-classifier fusion framework on Gabor images using a new fusion method called adaptive informative fusion scheme. Experimental results clearly show that this framework can greatly enhance the recognition performance.

The major contributions of this paper are:

1. Considering the specific property of the indoor and outdoor face recognition problem, we develop a novel algorithm called discriminant mutual subspace learning (DMSL) to improve the recognition performance.

2. Based on the developed DMSL algorithm, we further develop an integrated multi-classifier framework incorporating several techniques including an extended Gabor representation, DMSL, and a new fusion scheme called adap- 
tive informative fusion scheme to further boost the recognition performance.

3. The developed algorithms can also be used for other pattern recognition applications. For example, the DMSL algorithm can be used to address the generic inter-modality face recognition problem [16][17][18]; the proposed new fusion scheme is also a generic fusion method for combining the discriminant information from different classifiers.

\section{Discriminant Mutual Subspace Learning (DMSL)}

\subsection{Problem Formulation}

In our algorithm, each face is represented by a vector in a $d$-dimensional sample space $\mathcal{X}$. Suppose we are given a training set with $N_{I}$ indoor and $N_{E}$ outdoor face samples from $C$ different persons, denoted by $\left\{\left(\mathbf{x}_{1}^{(I)}, c_{1}^{(I)}\right),\left(\mathbf{x}_{2}^{(I)}, c_{2}^{(I)}\right), \ldots,\left(\mathbf{x}_{N_{I}}, c_{N_{I}}^{(I)}\right)\right\}$ and $\left\{\left(\mathbf{x}_{1}^{(E)}, c_{1}^{(E)}\right),\left(\mathbf{x}_{2}^{(E)}, c_{2}^{(E)}\right), \ldots,\left(\mathbf{x}_{N_{E}}, c_{N_{E}}^{(E)}\right)\right\}$ respectively. Here $c_{i}^{(I)}$ and $c_{i}^{(E)}$ are class labels of the corresponding indoor and outdoor samples.

The dimension of original face representation is very high, which incurs great computational difficulty and numerical instability in classification. Due to the highly structured nature of human faces, it is justifiable to assume that the face samples lie on a subspace with much lower dimension. In traditional subspace analysis methods, a projection matrix learned from the training set is applied to all samples to transform them to the subspace. Among various subspace methods, LDA[4] is among the most popular and widely used in face recognition literature, which aims at maximizing the ratio of between-class scatter matrix to within-class scatter matrix.

Though LDA shows its effectiveness in the face recognition with well-controlled condition, however, it works poorly for outdoor face images with significant illumination and environment changes. Considering that indoor and outdoor samples present greatly different characteristics, they should be separately modeled. In addition, considering that both the indoor and outdoor samples are associated to the same face object, there should be a discriminant mutual subspace reflecting the intrinsic face characteristics within the indoor and outdoor samples. Motivated by this rationale, we develop a novel subspace learning algorithm called Discriminant Mutual Subspace Learning. In the developed approach, we simultaneously learn two coupled transform matrices $\mathbf{W}_{I}$ and $\mathbf{W}_{E}$ for indoor and outdoor samples based on their mutual interactions. After the dual transforms are learned, the samples are projected to the mutual subspace by

$$
\mathbf{y}^{(I)}=\mathbf{W}_{I}^{T}\left(\mathbf{x}^{(I)}-\mathbf{m}^{(I)}\right), \mathbf{y}^{(E)}=\mathbf{W}_{E}^{T}\left(\mathbf{x}^{(E)}-\mathbf{m}^{(E)}\right),
$$

where $\mathbf{m}^{(I)}$ and $\mathbf{m}^{(E)}$ are the mean vectors of the indoor and outdoor samples respectively.

In addition, instead of using Euclidean distance, we employ magnitude of correlation to measure the similarities between an indoor sample $\mathbf{x}^{(I)}$ and an outdoor sample $\mathbf{x}^{(E)}$, which is written as

$$
s\left(\mathbf{x}^{(I)} ; \mathbf{x}^{(E)}\right)=\left(\mathbf{x}^{(I) T} \mathbf{x}^{(E)}\right)^{2} .
$$

For the purpose of discriminant learning, the learning objective of our method consists of two associative aspects: maximizing the similarity between indoor and outdoor samples from the same person; while minimizing the similarity between the samples from different persons. The two targets can be unified in the following form:

$\mathbf{W}_{I}, \mathbf{W}_{E}=\underset{\mathbf{W}_{I}, \mathbf{W}_{E}}{\operatorname{argmax}} \frac{\sum_{i=1}^{N_{I}} \sum_{j: c_{j}^{(E)}=c_{i}^{(I)}} s\left(\mathbf{W}_{I}^{T} \mathbf{x}_{i}^{(I)} ; \mathbf{W}_{E}^{T} \mathbf{x}_{j}^{(E)}\right)}{\sum_{i=1}^{N_{I}} \sum_{j: c_{j}^{(E)} \neq c_{i}^{(I)}} s\left(\mathbf{W}_{I}^{T} \mathbf{x}_{i}^{(I)} ; \mathbf{W}_{E}^{T} \mathbf{x}_{j}^{(E)}\right)}$

For conciseness of discussion, we convert the formulation into an equivalent form:

$\mathbf{W}_{I}, \mathbf{W}_{E}=\underset{\mathbf{W}_{I}, \mathbf{W}_{E}}{\operatorname{argmax}} \frac{\sum_{i=1}^{N_{I}} \sum_{j=1}^{N_{E}} r_{i j}^{(W)} s\left(\mathbf{W}_{I}^{T} \mathbf{x}_{i}^{(I)} ; \mathbf{W}_{E}^{T} \mathbf{x}_{j}^{(E)}\right)}{\sum_{i=1}^{N_{I}} \sum_{j=1}^{N_{E}} r_{i j}^{(B)} s\left(\mathbf{W}_{I}^{T} \mathbf{x}_{i}^{(I)} ; \mathbf{W}_{E}^{T} \mathbf{x}_{j}^{(E)}\right)}$

Here the coefficients $r_{i j}^{(I)}$ and $r_{i j}^{(E)}$ are defined as

$$
r_{i j}^{(W)}=\left\{\begin{array}{ll}
1 & \left(c_{i}^{(I)}=c_{j}^{(E)}\right. \\
0 & \left(c_{i}^{(I)} \neq c_{j}^{(E)}\right.
\end{array}\right), \quad r_{i j}^{(B)}=\left\{\begin{array}{cc}
1 & \left(c_{i}^{(I)} \neq c_{j}^{(E)}\right. \\
0 & \left(c_{i}^{(I)}=c_{j}^{(E)}\right.
\end{array}\right)
$$

\subsection{Learning by Optimization}

The transform matrices $\mathbf{W}_{I}$ and $\mathbf{W}_{E}$ can be learned by optimizing the objective function given in Eq.(4). To simplify our discussion, we first rewrite it in matrix form as follows:

$$
\begin{aligned}
\operatorname{Sim}_{W}\left(\mathbf{W}_{I}, \mathbf{W}_{E}\right) & =\sum_{i=1}^{N_{I}} \sum_{j=1}^{N_{E}} r_{i j}^{(W)} s\left(\mathbf{W}_{I}^{T} \mathbf{x}_{i}^{(I)} ; \mathbf{W}_{E}^{T} \mathbf{x}_{j}^{(E)}\right) \\
& =\sum_{i=1}^{N_{I}} \sum_{j=1}^{N_{E}} r_{i j}^{(W)}\left(\mathbf{x}^{(I) T} \mathbf{W}_{I} \mathbf{W}_{E}^{T} \mathbf{x}^{(E)}\right)^{2}
\end{aligned}
$$

Since, we have

$$
\begin{aligned}
& \left(\mathbf{x}^{(I) T} \mathbf{W}_{I} \mathbf{W}_{E}^{T} \mathbf{x}^{(E)}\right)^{2} \\
= & \operatorname{tr}\left(\mathbf{W}_{I}^{T} \mathbf{x}^{(I) T} \mathbf{x}^{(E)} \mathbf{W}_{E} \mathbf{W}_{E}^{T} \mathbf{x}^{(E) T} \mathbf{x}^{(I)} \mathbf{W}_{I}\right) \\
= & \operatorname{tr}\left(\mathbf{W}_{E}^{T} \mathbf{x}^{(E) T} \mathbf{x}^{(I)} \mathbf{W}_{I} \mathbf{W}_{I}^{T} \mathbf{x}^{(I) T} \mathbf{x}^{(E)} \mathbf{W}_{E}\right)
\end{aligned}
$$


Thus

$$
\begin{aligned}
& \operatorname{Sim}_{W}\left(\mathbf{W}_{I}, \mathbf{W}_{E}\right) \\
= & \operatorname{tr}\left(\sum_{i=1}^{N_{I}} \sum_{j=1}^{N_{E}} r_{i j}^{(W)} \mathbf{W}_{I}^{T} \mathbf{x}^{(I) T} \mathbf{x}^{(E)} \mathbf{W}_{E} \mathbf{W}_{E}^{T} \mathbf{x}^{(E) T} \mathbf{x}^{(I)} \mathbf{W}_{I}\right) \\
= & \operatorname{tr}\left(\sum_{i=1}^{N_{I}} \sum_{j=1}^{N_{E}} r_{i j}^{(W)} \mathbf{W}_{E}^{T} \mathbf{x}^{(E) T} \mathbf{x}^{(I)} \mathbf{W}_{I} \mathbf{W}_{I}^{T} \mathbf{x}^{(I) T} \mathbf{x}^{(E)} \mathbf{W}_{E}\right)
\end{aligned}
$$

Likewise, we have

$$
\begin{aligned}
& \operatorname{Sim}_{B}\left(\mathbf{W}_{I}, \mathbf{W}_{E}\right) \\
= & \operatorname{tr}\left(\sum_{i=1}^{N_{I}} \sum_{j=1}^{N_{E}} r_{i j}^{(B)} \mathbf{W}_{I}^{T} \mathbf{x}^{(I) T} \mathbf{x}^{(E)} \mathbf{W}_{E} \mathbf{W}_{E}^{T} \mathbf{x}^{(E) T} \mathbf{x}^{(I)} \mathbf{W}_{I}\right) \\
= & \operatorname{tr}\left(\sum_{i=1}^{N_{I}} \sum_{j=1}^{N_{E}} r_{i j}^{(B)} \mathbf{W}_{E}^{T} \mathbf{x}^{(E) T} \mathbf{x}^{(I)} \mathbf{W}_{I} \mathbf{W}_{I}^{T} \mathbf{x}^{(I) T} \mathbf{x}^{(E)} \mathbf{W}_{E}\right)
\end{aligned}
$$

Thus Eq.(4) can be expressed as

$$
\mathbf{W}_{I}, \mathbf{W}_{E}=\underset{\mathbf{W}_{I}, \mathbf{W}_{E}}{\operatorname{argmax}} \frac{\operatorname{Sim}_{W}\left(\mathbf{W}_{I}, \mathbf{W}_{E}\right)}{\left.\operatorname{Sim}_{I}, \mathbf{W}_{E}\right)}
$$

Recognizing the duality between $\mathbf{W}_{I}$ and $\mathbf{W}_{E}$ reflected in the formulas above. We devise an alternate optimization scheme which alternately optimizes $\mathbf{W}_{I}$ and $\mathbf{W}_{E}$ with the other fixed.

With $\mathbf{W}_{E}$ fixed, then we can construct

$$
\begin{aligned}
\mathbf{M}_{W}^{(I)} & =\sum_{i=1}^{N_{I}} \sum_{j=1}^{N_{E}} r_{i j}^{(W)} \mathbf{x}^{(I) T} \mathbf{x}^{(E)} \mathbf{W}_{E} \mathbf{W}_{E}^{T} \mathbf{x}^{(E) T} \mathbf{x}^{(I)}, \\
\mathbf{M}_{B}^{(I)} & =\sum_{i=1}^{N_{I}} \sum_{j=1}^{N_{E}} r_{i j}^{(B)} \mathbf{x}^{(I) T} \mathbf{x}^{(E)} \mathbf{W}_{E} \mathbf{W}_{E}^{T} \mathbf{x}^{(E) T} \mathbf{x}^{(I)}
\end{aligned}
$$

Thus $\mathbf{W}_{I}$ can be solved by following optimization problem:

$$
\mathbf{W}_{I}=\underset{\mathbf{W}_{I}}{\operatorname{argmax}} \frac{\operatorname{tr}\left(\mathbf{W}_{I}^{T} \mathbf{M}_{W}^{(I)} \mathbf{W}_{I}\right)}{\operatorname{tr}\left(\mathbf{W}_{I}^{T} \mathbf{M}_{B}^{(I)} \mathbf{W}_{I}\right)}
$$

This is a problem of maximizing Rayleigh quotient, which can be efficiently solved using eigen-decomposition as in LDA, and step-wise global optima is guaranteed.

Similarly, we can optimize $\mathbf{W}_{I}$ with $\mathbf{W}_{E}$ fixed as follows

$$
\mathbf{W}_{E}=\underset{\mathbf{W}_{E}}{\operatorname{argmax}} \frac{\operatorname{tr}\left(\mathbf{W}_{E}^{T} \mathbf{M}_{W}^{(E)} \mathbf{W}_{E}\right)}{\operatorname{tr}\left(\mathbf{W}_{E}^{T} \mathbf{M}_{B}^{(E)} \mathbf{W}_{E}\right)},
$$

where

$$
\begin{aligned}
\mathbf{M}_{W}^{(E)} & =\sum_{i=1}^{N_{I}} \sum_{j=1}^{N_{E}} r_{i j}^{(W)} \mathbf{x}^{(E) T} \mathbf{x}^{(I)} \mathbf{W}_{I} \mathbf{W}_{I}^{T} \mathbf{x}^{(I) T} \mathbf{x}^{(E)}, \\
\mathbf{M}_{B}^{(I)} & =\sum_{i=1}^{N_{I}} \sum_{j=1}^{N_{E}} r_{i j}^{(B)} \mathbf{x}^{(E) T} \mathbf{x}^{(I)} \mathbf{W}_{I} \mathbf{W}_{I}^{T} \mathbf{x}^{(I) T} \mathbf{x}^{(E)}
\end{aligned}
$$

\subsection{Discussions}

1. By using the developed dual transforms for indoor and outdoor samples, the different characteristics of indoor and outdoor images are explicitly taken into consideration. Hence, the learned model is more adapted to the indooroutdoor problem. The experiments will further validate the effectiveness of our method.

2. The derived procedure is step-wise globally optimal, i.e. with $\mathbf{W}_{I}$ fixed, the $\mathbf{W}_{E}$ obtained is global optima, and vice versa. Note that by employing correlation as similarity measurement, we can deduce the elegant optimization scheme introduced above without compromising the classification performance.

3. The formulation unifying $\mathbf{W}_{I}$ and $\mathbf{W}_{E}$ virtually embodies their duality. The optimization is in essence accomplished by their mutual interactions.

\section{Integrated Multi-classifier Fusion Frame- work}

The outdoor environment is subject to significant illumination change and other interferences, thus a single discriminant mutual subspace classifier may lack sufficient capability in modeling such complicated variations. To this end, we develop a multi-classifier fusion framework, which constructs multiple classifiers based on different features of face images, and then integrates them to form a sophisticated system. The structure of this framework is illustrated in Figure 1.

\subsection{Feature Description Using Extended Gabor Representation}

The Gabor wavelet has been shown to outperform the original appearance features in recent study [2][6]. It inherently possesses spatial locality and orientation selectivity. These properties endow the Gabor-based representation with capability in capturing the facial features of diverse scales and orientations and robustness to illumination and expression change. Wang and Tang [2] have shown that face recognition in an uncontrolled environment can substantially benefit from the Gabor features. However, in their work, the Gabor wavelet feature extraction is restricted in some fiducial points, thus the information contained in the Gabor representation is not sufficiently utilized. To overcome this problem, Li et. al. [11] proposed an extended Gabor representation which makes full use of the Gabor features of the whole image by convolving the image with 40 Gabor kernels (five different scales and eight different directions). Compared with the discrete landmark-based Gabor representation [6][2], the extended representation conveys more information and thus is more expressive. So in this work we will employ extended Gabor-based representation [11] in this paper. 
For each image, construct $\mathbf{4 0}$ Gabor images

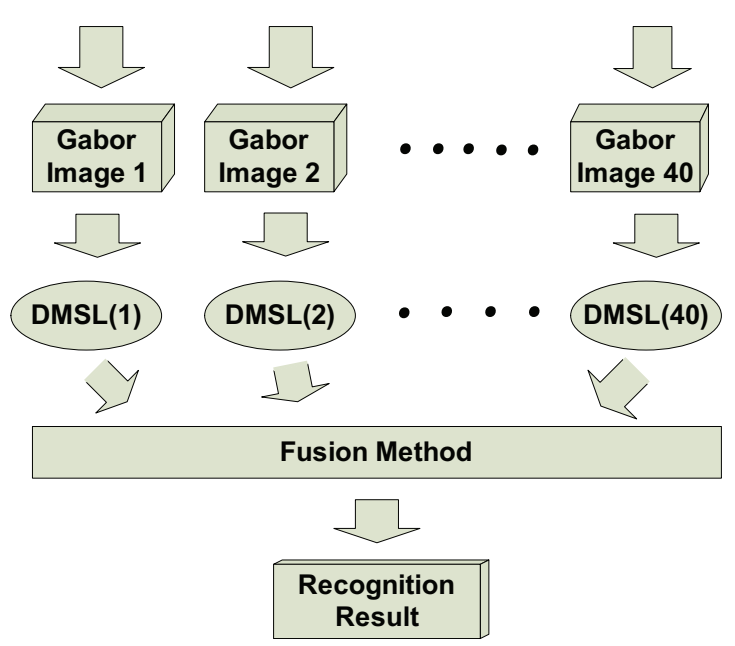

Figure 1. DMSL-based Multi-classifier Fusion Framework on Gabor Images

\subsection{DMSL-based Multi-classifier Fusion Framework}

By applying the extended Gabor representation [11], for each face, we can obtain an image sequence composing of 40 Gabor images. The Gabor images obtained by different kernels are similar to multiple frames in a video sequence, thus the fusion framework for video sequence developed in [14] can be applied here to improve the recognition accuracy and efficiency. Inspired by this idea, we propose a DMSL-based multi-classifier fusion framework with a novel fusion scheme developed. We first break the sequence into slices, with feature from each Gabor image as a slice. Then we apply DMSL to deal with each slice. Finally all the DMSL-based classifiers are combined into a final decision via a novel fusion scheme.

Previous researches have shown that combination of multiple classifiers can significantly improve accuracy and robustness of single classifier. There are a large number of fusion rules, such as the widely used majority voting rule and sum rule [12][13]. However, most of these fusion schemes are done in a heuristic manner, so the information that the ingredient classifiers convey is not fully utilized. In this section, we derive a novel classifier fusion scheme called adaptive informative fusion, which can adaptively weights more on confident classifiers to achieve the best performance for the final decision.

Suppose that there are $c$ classes $\left\{\mathcal{C}_{i}\right\}_{i=1}^{c}$ in total, and $n$ samples in the training set. $K$ classifiers are trained to clas- sify samples using DMSL to extract features. Denote the distance value between the sample $\mathrm{x}$ and the $\mathrm{i}$-th person in the $k$-th classifier space as $d_{k}^{i}(\mathbf{x})$. It is reasonable to assume that the random samples follow a normal distribution in the $k$-th classifier space, i.e.

$$
P_{k}\left(d_{k}^{i}(\mathbf{x}) \mid \mathcal{C}_{i}\right)=\frac{1}{\sqrt{2 \pi} \sigma} \exp \left(-\frac{\left(d_{k}^{i}(\mathbf{x})\right)^{2}}{2 \sigma^{2}}\right),
$$

where $\sigma$ is the standard deviation. According to the Bayesian rule, we derive the posterior probability

$$
P_{k}\left(\mathcal{C}_{i} \mid d_{k}^{i}(\mathbf{x})\right)=\frac{P_{k}\left(d_{k}^{i}(\mathbf{x}) \mid \mathcal{C}_{i}\right) P_{k}\left(\mathcal{C}_{i}\right)}{\sum_{j=1}^{c} P_{k}\left(d_{k}^{i}(\mathbf{x}) \mid \mathcal{C}_{j}\right) P_{k}\left(\mathcal{C}_{j}\right)},
$$

Usually each gallery class has the same prior probability, i.e. $P_{k}\left(\mathcal{C}_{1}\right)=\cdots=P_{k}\left(\mathcal{C}_{c}\right)$, so we have

$$
P_{k}\left(\mathcal{C}_{i} \mid d_{k}^{i}(\mathbf{x})\right)=\frac{P_{k}\left(d_{k}^{i}(\mathbf{x}) \mid \mathcal{C}_{i}\right)}{\sum_{q=1}^{c} P_{k}\left(d_{k}^{i}(\mathbf{x}) \mid \mathcal{C}_{j}\right)},
$$

which serves as an individual classifier.

It is commonly believed that it is impossible to find a classifier that can outperform others for all of the test samples. Some classifiers may perform better for a subset of test samples, while other classifiers may perform better for a different subset, although on average they may be very similar to each other in terms of overall accuracy. Hence it is desirable to design an adaptive weighting function to reflect each individual classifier's confidence on its decision accommodating a variety of test samples. Motivated by this rationale, we then define an adaptive weighting function below

$$
\mathcal{W}_{k}(\mathbf{x})=1-E_{k}(\mathbf{x})
$$

where $E_{k}(\mathbf{x})$ is the entropy of classes conditioned on test sample $\mathbf{x}$ in the $k$-th classifier space, defined by

$$
E_{k}(\mathbf{x})=\sum_{i=1}^{c} P_{k}\left(\mathcal{C}_{i} \mid d_{k}^{i}(\mathbf{x})\right) \log _{c} \frac{1}{P_{k}\left(\mathcal{C}_{i} \mid d_{k}^{i}(\mathbf{x})\right)}
$$

The combined decision rule is accordingly derived to classify sample $\mathbf{x}$ to the $i^{*}$ th class

$$
i^{*}=\arg \max _{i} \sum_{k=1}^{K} \mathcal{W}_{k}(\mathbf{x}) P_{k}\left(\mathcal{C}_{i} \mid d_{k}^{i}(\mathbf{x})\right),
$$

in which $P_{k}\left(\mathcal{C}_{i} \mid d_{k}^{i}(\mathbf{x})\right)$ denotes the posterior probability of test sample $\mathbf{x}$ belonging to the gallery class $\mathcal{C}_{i}$ in the $k$-th classifier space.

The adaptive weighting function has the following property. If posterior probabilities of test sample $\mathbf{x}$ belonging to all classes in the $k$-th classifier are equal, that means this classifier will fail to make any decision, then $E_{k}(\mathbf{x})=1$, i.e. $\mathcal{W}_{k}(\mathbf{x})=0$. On the contrary, if there is a classifier 
evaluating $P_{k}\left(\mathcal{C}_{i} \mid d_{k}^{i}(\mathbf{x})\right)=1$, which can definitely determine the class label of $\mathbf{x}$, then $E_{k}(\mathbf{x})=0$, i.e. $\mathcal{W}_{k}(\mathbf{x})=1$. By using such an adaptive weighting function, the highly confident individual classifier can be emphasized with good adaptation to the test data. In the experimental section, we will see the advantage of our fusion scheme over traditional fusion schemes.

Eventually, the procedure of our fusion method is summarized as follows.

In training stage,

1. For each training sample, obtain the Gabor-based image sequence composing of 40 Gabor images using the introduced Gabor feature description technique.

2. Construct DMSL-based classifiers based on Gabor images obtained with different Gabor kernels. There are 40 different kernels, so there are 40 DMSL-based classifiers.

In testing stage,

1. For each test sample, obtain the Gabor-based image sequence using the similar method as the training stage.

2. Use DMSL-based classifier to determine the classification respectively based on the corresponding Gabor image.

3. Combine the decisions made by DMSL-based classifiers with the new developed fusion scheme.

\section{Experiments}

Until now almost all face databases only contain photos captured under well-controlled indoor conditions and few have uncontrolled outdoor facial images. In order to systematically investigate this new research topic in face recognition, we first build a large mixed indoor and outdoor face database containing 1444 facial images from 62 distinct persons. The data size of this database is comparable to existing face databases for indoor face recognition. In this database, there are 23 persons with each one having 9 indoor images and 8 outdoor images, and 39 persons with each one having 15 indoor images and 12 outdoor images. So there are totally 1444 indoor and outdoor facial images with great indoor and outdoor discrepancies. For each person, we use three indoor images and three outdoor images as training data, and select one indoor image as reference sample and the remaining outdoor images as probe samples. There are totally 466 probe samples under uncontrolled outdoor conditions. To better evaluate the recognition performance we preprocess the face images through the following steps: 1) rotate the face images to align the vertical face orientation; 2) scale the face images so that the distances between the two eyes are the same for all images; 3 ) crop the face images to remove the background and the hair region; 4) apply histogram equalization for photometric normalization.

We first compare our discriminant mutual subspace learning $(D M S L)$ algorithm with conventional subspace methods: Eigenface [3], Fisherface [4], Bayesian method [5], and Unified subspace analysis [2] on the traditional Gabor features, as shown in Table 1. The poor results of the traditional subspace methods confirm that the traditional subspace models are not suitable for the indoor and outdoor recognition problem due to the great discrepancies between the two styles of images. It is encouraging to see that our method, discriminant mutual subspace learning, significantly outperforms all the traditional subspace methods by a large margin.

Table 1. Comparison of recognition results on the traditional Gabor features.

\begin{tabular}{|c|c|}
\hline Recognition methods & Recognition Rate \% \\
\hline \hline PCA & 28.97 \\
\hline LDA & 75.11 \\
\hline Bayesian & 75.54 \\
\hline Unified subspace analysis & 80.26 \\
\hline Discriminant mutual subspace learning & 87.98 \\
\hline
\end{tabular}

Table 2. Comparison of recognition results on the extended Gabor features.

\begin{tabular}{|c|c|}
\hline Recognition methods & Recognition Rate \% \\
\hline $\begin{array}{c}\text { Unified subspace analysis } \\
\text { based multi-classifier } \\
\text { framework using sum rule }\end{array}$ & 85.41 \\
\hline $\begin{array}{c}\text { Unified subspace analysis } \\
\text { based multi-classifier } \\
\text { framework using voting rule }\end{array}$ & 86.05 \\
\hline $\begin{array}{c}\text { Unified subspace analysis } \\
\text { based multi-classifier } \\
\text { framework using our fusion scheme }\end{array}$ & 89.06 \\
\hline $\begin{array}{c}\text { DMSL-based multi-classifier } \\
\text { framework using sum rule }\end{array}$ & 93.56 \\
\hline $\begin{array}{c}\text { DMSL-based multi-classifier } \\
\text { framework using voting rule }\end{array}$ & 94.64 \\
\hline $\begin{array}{c}\text { DMSL-based multi-classifier } \\
\text { framework using our fusion scheme }\end{array}$ & 96.78 \\
\hline
\end{tabular}

In the second experiment, we investigate the performance of our proposed multi-classifier fusion framework on the extended Gabor features (Gabor images) under different fusion schemes, and also compare the DMSL-based multiclassifier fusion framework and the unified subspace analysis based multi-classifier fusion framework. In our multiclassifier framework, 40 Gabor wavelet images are acquired for each facial image, accordingly 40 subspace classifiers are constructed. Then the generated multiple classifiers are combined into a final decision via a fusion rule. In this experiment, three kinds of fusion rules are used here, including the adaptive fusion rule and the two widely used traditional fusion rules (sum rule and majority rule). The comparative results are summarized in Table 2. From the results, 
we have the following observations. Firstly, compared with the results in Table 1, the developed multi-classifier fusion framework further boosts the recognition performance, regardless of which subspace method is used. This clearly shows the advantage of our fusion framework. Secondly, the DMSL-based multi-classifier fusion framework outperforms the unified subspace analysis based multi-classifier fusion framework. Thirdly, among the three kinds of fusion methods, the new fusion scheme achieves the best performance. This shows the superiority of this new fusion scheme over the traditional fusion methods such as sum rule and voting rule. Finally, by incorporating all the new algorithms into this framework, we achieve the best recognition performance, $96.78 \%$. Considering the great difficulty of this problem, this result is very encouraging and clearly validates the effectiveness of our framework.

\section{Conclusions}

In this paper, we develop a novel algorithm called discriminant mutual subspace learning (DMSL) for the challenging problem of indoor and outdoor face recognition. Different from the conventional subspaces methods that simply train a subspace model based on the face space for classification, our approach first learns two associative transforms for indoor and outdoor samples respectively and then find the discriminant mutual subspace for classification. Moreover, to enhance the performance of DMSL, we further develop a DMSL-based multi-classifier framework by integrating several novel techniques including extended Gabor representation, DMSL, and adaptive informative fusion scheme. Extensive experiments clearly show the effectiveness of our new algorithms on a large indoor-outdoor face database.

\section{Acknowledge}

The work described in this paper was fully supported by grants from the Research Grants Council of the Hong Kong Special Administrative Region.

\section{References}

[1] P. J. Phillips, P. Grother, R. J. Micheals, D. M. Blackburn, E. Tabassi, and M. Bone, "Face Recognition Vendor Test: Evaluation Report," March 2003.

[2] X. Wang and X. Tang, "Improving indoor and outdoor face recognition using unified subspace analysis and gabor features," IEEE International Conference on Image Processing, 2004.

[3] M. Turk and A. Pentland, "Face recognition using eigenfaces," IEEE International Conference on Computer Vision and Pattern Recognition, pp. 586-591, 1991.
[4] V. Belhumeur, J. Hespanda, and D. Kiregeman, "Eigenfaces vs. fisherfaces: Recognition using class specific linear projection," IEEE Trans. on PAMI, Vol. 19, No. 7, pp. 711-720, July 1997.

[5] B. Moghaddam, T. Jebara, and A. Pentland, "Bayesian face recognition," Pattern Recognition, Vol. 33, pp. 1771-1782, 2000.

[6] L. Wiskott, J.M. Fellous, N. Kruger, and C. von der Malsburg, "Face Recognition by Elastic Bunch Graph Matching," IEEE Trans. on PAMI, Vol. 19, No.7, pp. 775-779, July, 1997.

[7] X. Wang and X. Tang, "Unified Subspace Analysis for Face Recognition," Proceedings of ICCV, pp. 679-686, Oct. 2003.

[8] S. Nayar, and H. Murase, "Dimensionality of Illumination Manifolds in Eigenspace," Technical Report CUCS-021-94, Dept. of Computer Science, Columbia University, 1994.

[9] R. Basri and D. Jacobs, "Lambertian Reflectance and Linear Subspaces," IEEE Trans. on PAMI, Vol. 25, No. 2, 2003.

[10] A. S. Georghiades, P. N. Belhumeur, and D. J. Kriegman, "From Few to Many: Illumination Cone Models for Face Recognition under Variable Lighting and Pose," IEEE Trans. on PAMI, Vol. 23, No. 6, pp. 643-660, June 2001.

[11] Z. Li, W. Liu, D. Lin, and X. Tang, "Nonparametric Subspace Analysis for Face Recognition," IEEE International Conference on Computer Vision and Pattern Recognition, Vol. 23, No. 6, pp. 643-660, June 2001.

[12] L. Xu, A. Krzyzak, and C. Y. Suen, "Method of Combining Multiple Classifiers and Their Applications to Handwriting Recognition," IEEE Trans. on System, Man, and Cybernetics, Vol. 22, No. 3, pp. 418-435, 1992.

[13] T. K. Ho, J. Hull, and S. Srihari, "Decision Combination in Multiple Classifier Systems," IEEE Trans. on PAMI, Vol. 16, No. 1, pp. 66-75, Jan. 1994.

[14] X. Tang and Z. Li, "Frame synchronization and multilevel subspace analysis for video based face recognition," Prof. IEEE Conf. Computer Vision and Pattern Recognition (CVPR), June 2004.

[15] X. Wang and X. Tang, "A unified framework for subspace face recognition," IEEE Trans. Pattern Analysis and Machine Intelligence, vol. 26, no. 9, pp. 1222-1228, Sep. 2004.

[16] X. Tang and X. Wang, "Face sketch synthesis and recognition," Proc. Ninth IEEE Int'l Conf. on Computer Vision, pp. 687-694, Oct. 2003.

[17] X. Tang and X. Wang, "Face sketch recognition," IEEE Trans. on Circuits and Systems for Video Technology, 14(1), pp. 50-57, 2004.

[18] Dahua Lin and Xiaoou Tang. "Inter-Modality Face Recognition," Proceedings of 9th IEEE European Conference on Computer Vision, Part I, Pages:13-26, 2006. 